\title{
An Experimental Evaluation of a 3D Visible Light Positioning System in an Industrial Environment with Receiver Tilt and Multipath Reflections
}

\author{
Yousef Almadani ${ }^{1 *}$, Muhammad Ijaz ${ }^{1}$, Bamidele Adebisi ${ }^{1}$, Sujan \\ Rajbhandari $^{2}$, Sander Bastiaens ${ }^{3}$, Wout Jospeh ${ }^{3}$, and David Plets ${ }^{3}$ \\ ${ }^{1}$ Manchester Metropolitan University, Faculty of Science and Engineering, Department \\ of Engineering, Manchester, M1 5GD, United Kingdom \\ ${ }^{2}$ Huawei Technologies Sweden AB, 41250 Gothenburg, Sweden \\ ${ }^{3}$ Ghent University, imec-WAVES, Department of Information Technology, \\ iGent-Technologiepark 126, Ghent, 9052, Belgium
}

\begin{abstract}
In this paper, two different three-dimensional (3D) indoor visible light positioning (VLP) algorithms are experimentally assessed for an industrial environment. The Cayley-Menger determinant (CMD) and linear least square (LLS) trilateration algorithms use the received signal strength (RSS) to estimate the receiver's 3D position without prior knowledge of its height. The unknown 3D position of the receiver is estimated by the trilateration algorithms coupled with a cost function under different realistic scenarios. The performances of the algorithms are experimentally evaluated in terms of positioning error by considering two different light-emitting diode (LED) configurations in the presence of different receiver tilt angles, and with multipath reflections. It is observed that the widespread square LED configuration results in position ambiguities while a star-shaped configuration is much more accurate. Experimental tests performed in a $4 \mathrm{~m} \times 4 \mathrm{~m} \times 4.1 \mathrm{~m}$ area with four LEDs reported a median positioning error of 10.6 and $10.5 \mathrm{~cm}$ using the LLS and CMD algorithms, respectively, without the presence of receiver tilt or multipath reflections. However, when a receiver tilt of $10^{\circ}$ was added, the median error increased to $22.7 \mathrm{~cm}$ using the LLS algorithm and $21.6 \mathrm{~cm}$ using the CMD algorithm. Overall, the achieved mean and maximum values using the LLS algorithm were 13.1 and $39 \mathrm{~cm}$, respectively, while they were 12.2 and $34 \mathrm{~cm}$ using the CMD algorithm.
\end{abstract}


Key words: indoor visible light positioning, localization, industrial, tilt, VLC, VLP

\section{Introduction}

Indoor positioning is a very promising research domain that is gaining wide attention due to its potential in Industry 4.0 and the health sector. Conventional positioning methods that rely on satellites such as global positioning system (GPS) are unreliable for indoor positioning due to the high penetration loss from walls and building materials. Complementary methods such as assisted-GPS and pseudo-satellite have been proposed to address the shortcomings of conventional satellite-based systems. However, the accuracies of these systems are still inadequate with the added complexity of integrating two different systems [1]. Other technologies have also been proposed for indoor positioning and navigation such as Bluetooth, ultrasound, ultrawideband (UWB), and radio-frequency (RF) based techniques [2]. While encouraging results have been achieved using Bluetooth and UWB, there is also another emerging technology that makes use of the ubiquitous light fixture's infrastructure.

Visible light positioning (VLP) is one of the most promising technologies being proposed for indoor positioning given the readily available lighting infrastructure and its many advantages such as increased bandwidth, security and low relative complexity when compared with RF-based positioning. While most of the technologies being researched and proposed for indoor localization are based on the highly congested RF spectrum, VLP systems are not sensitive to electromagnetic interference, which enables them to be used in areas that are sensitive to electromagnetic waves such as hospitals and certain power plants [3].

\section{Related Work}

In [4], the researchers proposed a multiple-classifiers fusion localization framework by using received signal strength (RSS) fingerprints. The experiment was performed within a $0.7 \mathrm{~m} \times 0.7 \mathrm{~m}$ area with four LEDs and achieved a median square positioning error of less than $5 \mathrm{~cm}$ for the majority of the area. In [5], a 3D VLC positioning system based on modified particle swarm optimization (PSO) algorithm is presented and has been experimentally tested. The researchers evaluated the system using four LEDs in a cube 
frame measuring $0.9 \mathrm{~m} \times 0.9 \mathrm{~m} \times 1.5 \mathrm{~m}$ and achieved an average error of $3.5 \mathrm{~cm}$ for a 3D VLP system. In [6], a machine learning (ML) technique with height tolerance was tested using three LEDs within an area of $1.1 \mathrm{~m} \times 1 \mathrm{~m}$ $\times 2.5 \mathrm{~m}$. The result shows that over $80 \%$ of the results can be under $5 \mathrm{~cm}$ with an improved height tolerance range of $15 \mathrm{~cm}$. Researchers in [7] introduced and experimentally tested a VLP method based on median shift (MS) algorithm and unscented Kalman filter (UKF) using image sensors. The test area of their experimental setup was $1.9 \mathrm{~m} \times 1 \mathrm{~m} \times 1.9 \mathrm{~m}$ and achieved a positioning accuracy of up to $0.42 \mathrm{~cm}$, with an accuracy of $1.41 \mathrm{~cm}$ when half of the LED was shielded. The work in [8] used an RSS-based VLP system combined with a deep neural network based on the Bayesian Regularization (BR-DNN) with a sparse diagonal training data set. The method was tested in a $1.8 \mathrm{~m} \times 1.8 \mathrm{~m} \times 2.1 \mathrm{~m}$ area and achieved a maximum positioning error of $4.58 \mathrm{~cm}$ for an even set, and $3.4 \mathrm{~cm}$ under a diagonal set of LEDs. In [9], a low-complexity time-difference-of-arrival (TDoA) method with an enhanced practical localization using cross-correlation is reported and achieved a positioning accuracy of $9.2 \mathrm{~cm}$ in a $1.2 \mathrm{~m} \times 1.2 \mathrm{~m}$ testbed area. A $2 \mathrm{D}$ VLP system using differential phase difference of arrival (DPDoA) was experimentally tested in [10] and achieved an average root-mean-square (RMS) positioning error of $1.8 \mathrm{~cm}$ and a maximum of $8 \mathrm{~cm}$ in a testbed area of $1 \mathrm{~m} \times 1.2 \mathrm{~m} \times 2 \mathrm{~m}$. Researchers in [11] proposed a fusion positioning system based on extended Kalman filters (EKF), which uses an inertial navigation unit to improve the performance of the VLP system. An average positioning error of $33.9 \mathrm{~cm}$ was achieved based on RSS alone and $14.5 \mathrm{~cm}$ when combined with an EKF.

Three typical office environments were tested in [12]. Their proposed method locates the receiver using trilateration/multi-lateration if over three light sources are perceived, along with an optimization process. If less than three signals are received, then a fusion method is used with an inertial measurement unit (IMU). The achieved $90^{\text {th }}$ percentile positioning errors for the three environments were $0.4 \mathrm{~m}, 0.7 \mathrm{~m}$, and $0.8 \mathrm{~m}$. When only one transmitter is available, the $90^{\text {th }}$ percentile error increased to $1.1 \mathrm{~m}$. The work in [13] proposed the use of the received light intensity with accelerometer measurements to compute distances between the transmitters and the receiver. An error of less than $25 \mathrm{~cm}$ was reported in a $5 \mathrm{~m} \times 3 \mathrm{~m} \times 3 \mathrm{~m}$ area. A gain difference positioning method based on the angle of arrival and the received signal strength was proposed in [14]. The method uses multiple tilted receivers to calculate the 3D location with reported average error distances of 
Table 1: A summary of the experimental work in indoor VLP systems

\begin{tabular}{|c|c|c|c|c|c|}
\hline Ref. & Method & $2 \mathrm{D} / 3 \mathrm{D}$ & Test Area (W L H) (m) & Accuracy $(\mathrm{cm})$ & No. of LEDs \\
\hline$[4]$ & Fingerprints & $2 \mathrm{D}$ & $0.7 \times 0.7 \times 1.48$ & 5 & 4 \\
\hline [6] & RSS w/ ML & $3 \mathrm{D}$ & $1.1 \times 1 \times 2.5$ & 3.65 & 3 \\
\hline [7] & MS-UKF & $2 \mathrm{D}$ & $1.9 \times 1 \times 1.9$ & 0.42 & 4 \\
\hline [8] & RSS w/ BR-DNN & $2 \mathrm{D}$ & $1.8 \times 1.8 \times 2.1$ & 4.58 & 4 \\
\hline [9] & TDoA & $2 \mathrm{D}$ & $1.2 \times 1.2 \times 2$ & 9.2 & 3 \\
\hline$[10]$ & DPDoA & $2 \mathrm{D}$ & $1 \times 1.2 \times 2$ & 1.8 & 3 \\
\hline$[11]$ & $\begin{array}{c}\text { RSS } \\
\text { RSS-EKF }\end{array}$ & $2 \mathrm{D}$ & $2.5 \times 2.84 \times 2.5$ & $\begin{array}{l}33.9 \\
14.5\end{array}$ & 7 \\
\hline [13] & RSS w/ Accelerometer & $3 \mathrm{D}$ & $5 \times 3 \times 3$ & 25 & 3 \\
\hline [14] & RSS ratio & $3 \mathrm{D}$ & $2 \times 2 \times 2.5$ & 3 & $1 \mathrm{w} /$ multiple PDs \\
\hline [5] & PSO & $3 \mathrm{D}$ & $0.9 \times 0.9 \times 1.5$ & 3.492 & 4 \\
\hline [15] & LED-ID w/ ROS & $2 \mathrm{D}$ & $1 \times 1 \times 1.5$ & 0.82 & 4 \\
\hline$[16]$ & LED-ID w/ ROS \& ML & $2 \mathrm{D}$ & $\begin{array}{c}0.8 \times 0.8 \times 2 \\
5 \times 8\end{array}$ & $\begin{array}{c}2 \\
45\end{array}$ & 5 \\
\hline [12] & RSS w/ IMU & $3 \mathrm{D}$ & $\begin{array}{c}2 \times 12 \\
3.5 \times 6.5\end{array}$ & $\begin{array}{l}70 \\
80\end{array}$ & 5 \\
\hline
\end{tabular}

${ }_{1}$ less than $3 \mathrm{~cm}$. Table 1 provides a summary of the discussed experimental 72 work on indoor VLP systems.

In [15], the researchers proposed an indoor robot VLP positioning package based on robot positioning system (ROS) with a efficient LED-ID detection scheme for rolling shutter. The system was experimentally tested in a $1 \mathrm{~m}$ $\times 1 \mathrm{~m} \times 1.5 \mathrm{~m}$ area with 36 uniformly distributed test points. The results reported an average accuracy of $0.82 \mathrm{~cm}$, while $90 \%$ of the errors were less than $1.417 \mathrm{~cm}$. The work in [16] proposed a double light positioning algorithm. The system uses LED-ID to determine the position of a receiver as well as a CMOS image sensor combined with machine learning a algorithm to identify the LED-ID. The system was tested in a $0.8 \mathrm{~m} \times 0.8 \mathrm{~m} \times 2 \mathrm{~m}$ area and all of the reported positioning errors were within $3.85 \mathrm{~cm}$ with an average accuracy of $2 \mathrm{~cm}$

As can be seen, the majority of the experimental work studied the performance of 2D VLP systems and generally required the use of additional hardware or the use of some complex algorithm for 3D localization. Additionally, most of the experiments analyzed the performance in relatively very small areas. In contrast to some of the previous works by other researchers, this paper examines a purely RSS-based 3D VLP system in a higher and larger area without the need for an additional receiver or complex algorithms.

In this paper, we experimentally assess and compare the performances of two 3D VLP positioning algorithms under different scenarios that are realistic industrial environments. The Cayley-Menger determinant (CMD) 
and linear least square (LLS) algorithms are coupled with a cost function to estimate a true 3D position without prior knowledge of the receiver's height. The algorithms are evaluated for two different LED configurations with different degrees of receiver tilt, and in the presence of a filled storage rack to examine the effect of multipath reflections on the performance. The algorithm could be used for VLP-based unmanned aerial vehicles (UAVs) tracking in industrial warehouses. This is an emerging area where UAVs, or drones, are employed for different sets of application such as stock-taking in warehouses and inspecting hard-to-reach areas [17]. The commonly used RF-based technologies generally suffer from electromagnetic interference or unstable RF signals, deeming it unsuitable in providing high positioning accuracy. It is especially not suitable in environments that have constant sudden changes, e.g. forklifts or automated guided vehicles (AGVs), and movement of people. This paper is partly an extension of our previous work in [17]. However, this work considers an additional LED layout configuration, an additional receiver tilt angle value, considers the presence of storage rack, and examines the performance for $2 \mathrm{D}$ positioning as well.

The remainder of the paper is organized as follows: Section 3 details the experimental setup. Section 4 presents the system model and the positioning algorithms along with the cost function. Experimental results are presented in Section 5 and is then followed by a discussion of the main findings in Section 6. Finally, the paper concludes in Section 7.

\section{Experimental Setup}

The 3D algorithm is analyzed experimentally in a VLP lab that measures $4 \mathrm{~m} \times 4 \mathrm{~m}$ with the height of the LEDs at approximately $4.1 \mathrm{~m}$, as shown in Figure 1 (a). Black curtains are used as a substitute for walls to ensure that uncontrolled reflections from walls and objects are avoided. Four BXRE-50C3001-D-24 LEDs, shown in the inset of Figure 1 (a), are intensity-modulated using transmitting pulse trains with a duty cycle of 0.5 with frequencies of $500 \mathrm{~Hz}, 1 \mathrm{kHz}, 2 \mathrm{kHz}$, and $4 \mathrm{kHz}$. This ensures that the contributions from the different LEDs can be demultiplexed individually at the receiver's side.

The receiver is a commercial photodiode with an integrated electrical amplifier (PDA36A2 ${ }^{1}$ by Thorlabs) that has an active area $A_{p d}$ of $13 \mathrm{~mm}^{2}$.

\footnotetext{
${ }^{1}$ https://www.thorlabs.com/thorproduct.cfm?partnumber=PDA36A2
} 


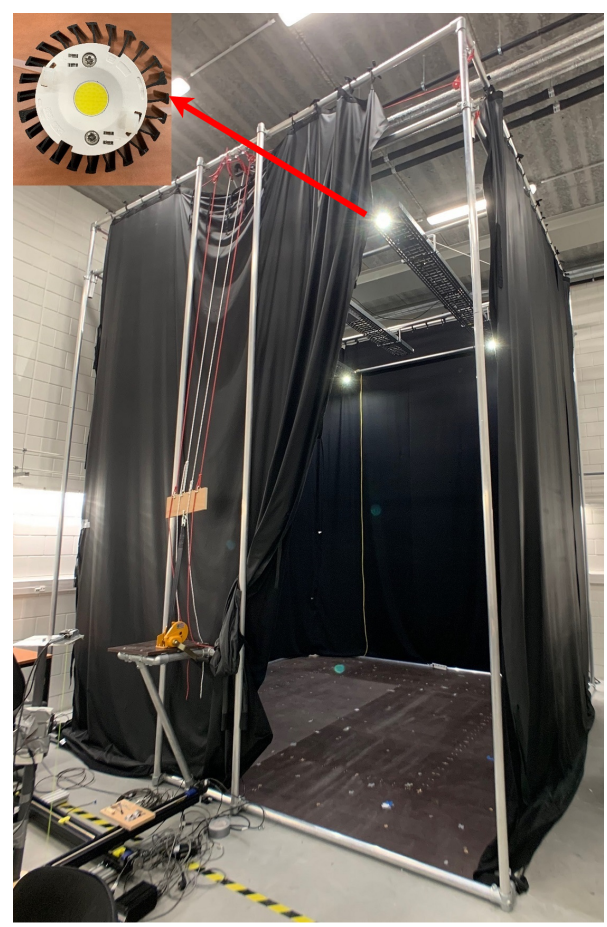

(a)

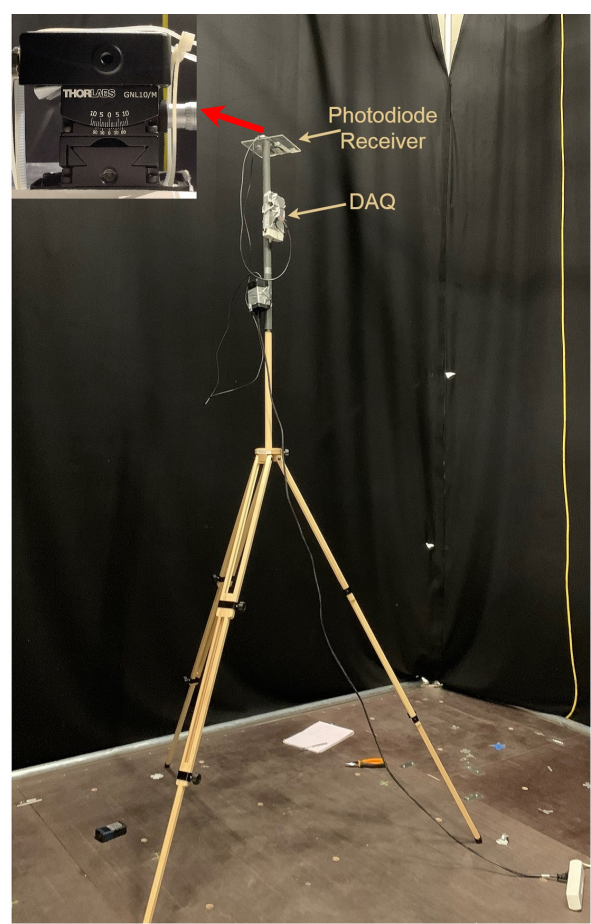

(b)

Figure 1: (a) The VLP lab experimental setup with black curtains with a view of the LEDs attached to ceiling rails, and (b) a tripod with the receiver mounted on top.

The photodiode's responsivity was estimated at $0.22 \mathrm{~A} / \mathrm{W}$ by weighing the photodiode's responsivity spectrum with the LED's spectrum. The receiver is attached to a tripod with a vertical pole that allows adjustment of the receiver's height as shown in Figure 1 (b). The data is acquired using National Instrument's USB-6212 for processing. A fast Fourier transform (FFT)-based demodulation is used to extract the received power values for each LED in MATLAB ${ }^{\circledR}$, as specified in [18]. Table 2 shows the main parameters used in the experimental setup.

Figure 2 shows a path consisting of forty-eight points selected to take the receiver around the room at different heights ranging from $0.64 \mathrm{~m}$ to $2.55 \mathrm{~m}$. The black line indicates the travel path, the green square denotes the start point, and red denotes the endpoint. The measurements were configured to sample 256 times using the DAQ with a sampling rate of $128 \mathrm{kHz}$. Twenty- 


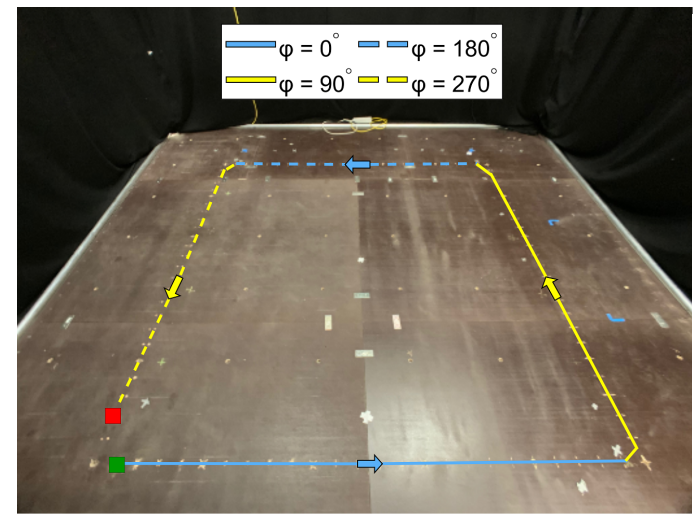

(a)

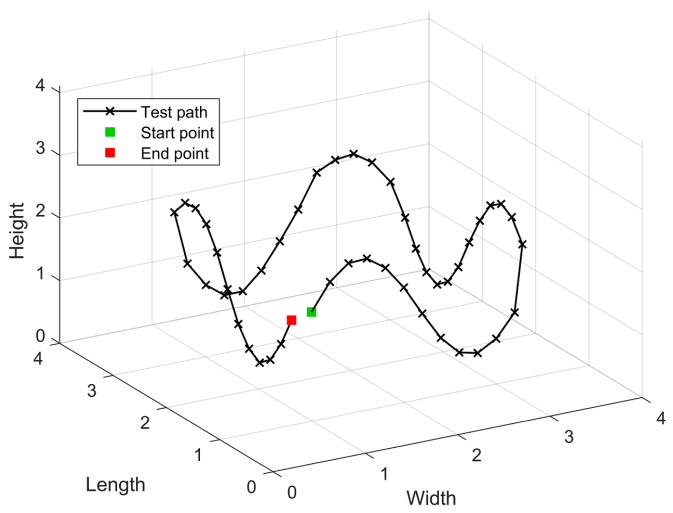

(b)

Figure 2: (a) The test path shown inside the VLP lab demonstrating the azimuthal orientation $\varphi$ of the receiver; (b) A 3D view of the path demonstrating the height variations of the receiver along the specified path.

five power value readings were averaged at each location to reduce the impact of noise.

Two LED configurations denoted as 'Square' and 'Star' are used for the evaluation of the VLP as shown in Figure 3. The square-shaped is a typical configuration that is adopted by many researchers while the star configuration has a central LED circularly surrounded by the other three LEDs. Our previous work in [19] indicates that a classic configuration with four LEDs mounted in a square-shape is not able to accurately solve the 3D position ambiguity. Therefore, to counter this problem, a star-shaped configuration was proposed.

\section{System Model}

In this section, the VLC's system model is outlined and the positioning algorithms along with the cost function are explained.

\subsection{VLC System Model}

The radiation of an LED chip follows a Lambertian pattern. Considering the line-of-sight (LoS) path between the LED transmitters and the receiver, 


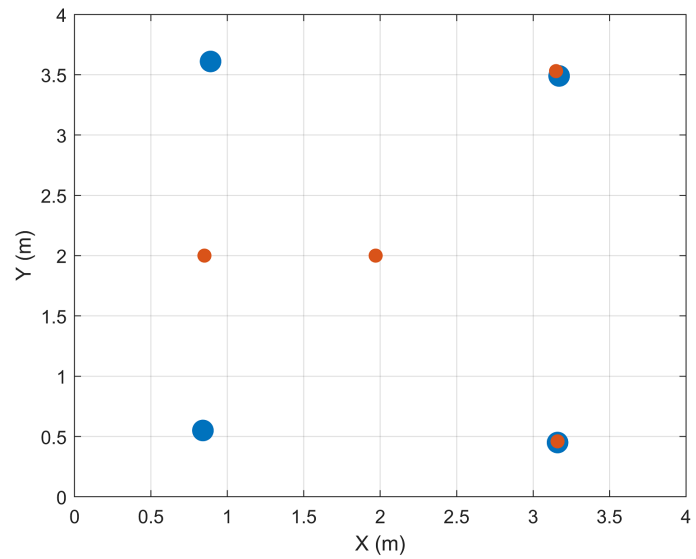

Figure 3: Top view of LEDs' locations in the with the blue dots representing the 'Square' configuration and the red dots representing the 'Star' configuration. heceived power can be modeled as [20]:

$$
P_{r i}=P_{t i} \frac{(m+1) A_{p d}}{2 \pi d_{i}^{2}} \cos ^{m}(\alpha) \cos (\beta) T_{p d}(\beta) G_{p d}(\beta)
$$

where $P_{t i}$ is the transmitted power from the $i^{t h}$ LED, $m$ is the Lambertian order, $d_{i}$ is the distance between the $i^{\text {th }}$ LED transmitter and the receiver, $\alpha$ is the angle of irradiance, $\beta$ is the angle of incidence. The parameters are illustrated in Figure 4 (a). The optical filter's gain $T_{p d}(\beta)$, and the optical concentrator's gain $G_{p d}(\beta)$ are assumed to be equal to 1. Additionally, by assuming that the transmitters and the receiver are horizontally parallel, $\cos (\alpha)=\cos (\beta)=\frac{h_{L E D}-z}{d_{i}}=\frac{\Delta h}{d_{i}}$, then $d_{i}$ can be estimated as $\widehat{d}_{i}$ using the received signal power, $\stackrel{d}{P}_{r i}[21]$ :

$$
\widehat{d}_{i}=\sqrt[m+3]{\frac{(m+1) A_{p d} P_{t i} \Delta h^{m+1}}{2 \pi P_{r i}}}
$$

where $\Delta h=h_{L E D}-z$ is the unknown vertical height difference between the $\mathrm{LED}_{i}$ transmitter and the receiver. Since $\Delta h$ is unknown, the estimated distance $\widehat{d}_{i}$ cannot be directly calculated from $P_{r i}$ without knowing $\Delta h$, or equivalently, $z$. Due to this, a set of estimated distances $\widehat{d}_{i}$ is generated for different receiver heights, $z$, ranging from a minimum height $h_{\min }$ to maximum height, $h_{\max } \leq h_{L E D}$ with $1 \mathrm{~mm}$ intervals. 
Table 2: Summary of the system parameters

\begin{tabular}{cc}
\hline Parameter & Value \\
\hline Room Width x Length x Height & $4 \mathrm{~m} \times 4 \mathrm{~m} \times 4.1 \mathrm{~m}$ \\
Transmitters' Power $-P_{t}$ & $13.3 \mathrm{~W}, 16.6 \mathrm{~W}, 16.4 \mathrm{~W}, 16.1 \mathrm{~W}$ \\
Transmitter's semi-angle $-\alpha$ & $60^{\circ}$ \\
Receiver's Height Range $-z$ & $0.64-2.55 \mathrm{~m}$ \\
Photodetector's Area $-A_{p d}$ & $13 \mathrm{~mm}^{2}$ \\
Receiver's Responsivity & $0.22 \mathrm{~A} / \mathrm{W}$ \\
\hline
\end{tabular}

172

The measured power of the LEDs can vary from their advertised values by up to $20 \%$, as demonstrated in $[22,23]$. Due to this, we collect one measurement directly under each transmitter as a calibration step $(\alpha=\beta=$ $0)$. Then the estimated transmitted power is calculated using $P_{t i}=\frac{P_{r i} 2 \pi d_{i}^{2}}{A_{p d}(m+1)}$ [24]. Table 2 lists the transmitted power for each transmitter.

In the case of receiver tilt, the received power will be impacted by an adapted angle of incidence. In this case, the angle of incidence in (1) is replaced with:

$$
\cos \left(\beta_{\text {tilt }}\right)=\frac{\left(x-x_{i}\right) \cos (\varphi) \sin (\theta)+\left(y-y_{i}\right) \sin (\varphi) \sin (\theta)+\left(z-h_{L E D}\right) \cos (\theta)}{d}
$$

where $(x, y, z)$ are the receiver's coordinates, $\left(x_{i}, y_{i}, z_{i}\right)$ are the LED's coordinates, $\theta$ is the receiver's tilting angle, which is the angle difference between the normal vector of the xy-plane and the normal vector of the receiver. $\varphi$ is the azimuthal rotation angle, which is the angle difference between the $\mathrm{x}$-axis and the orthogonal projection of the receiver's normal vector on the xy-plane.

\subsection{Positioning Algorithms}

Two positioning algorithms are used in this paper, CMD and LLS. The performance of the CMD algorithm is compared with LLS as the latter is widely adopted in VLP systems.

\subsubsection{Cayley-Menger Determinant}

The Cayley-Menger determinant is used in distance geometry for determining the volume of a triangular pyramid (tetrahedron) based on the distances between any two of four vertices [25]. Figure 4 (b) shows the position 
of three points (transmitters), $p 1, p 2$, and $p 3$, with $p 4$ being the unknown receiver's location.

The Cayley-Menger bideterminant of two sequences of $n$ points $\left[p_{1}, p_{2}, \ldots, p_{n}\right]$ and $\left[q_{1}, q_{2}, \ldots, q_{n}\right]$ is defined as $[26]$ :

$$
D\left(p_{1}, \ldots, p_{n} ; q_{1}, \ldots, q_{n}\right)=2\left(\frac{-1}{2}\right)^{n}\left|\begin{array}{ccccc}
0 & 1 & 1 & 1 & 1 \\
1 & D\left(p_{1}, q_{1}\right) & D\left(p_{1}, q_{2}\right) & \cdots & D\left(p_{1}, q_{n}\right) \\
1 & D\left(p_{2}, q_{1}\right) & D\left(p_{2}, q_{2}\right) & \cdots & D\left(p_{2}, q_{n}\right) \\
\vdots & \vdots & \vdots & \ddots & \vdots \\
1 & D\left(p_{n}, q_{1}\right) & D\left(p_{n}, q_{2}\right) & \cdots & D\left(p_{n}, q_{n}\right)
\end{array}\right|
$$

where $D\left(p_{i}, q_{j}\right)$ is the squared distance between points $p_{i}$ and $q_{j}$. When two sequences of points are the same (i.e., $\left.p_{i}=q_{i}\right)$, then $D\left(p_{1}, \ldots, p_{n} ; q_{1}, \ldots, q_{n}\right)$ is denoted by $D\left(p_{1}, \ldots, p_{n}\right)$ and is simply called CMD [26]. So (4) becomes:

$$
D\left(p_{1}, p_{2}, p_{3}, p_{4}\right)=\left(\frac{1}{8}\right)\left|\begin{array}{ccccc}
0 & 1 & 1 & 1 & 1 \\
1 & 0 & D\left(p_{1}, p_{2}\right) & D\left(p_{1}, p_{3}\right) & D\left(p_{1}, p_{4}\right) \\
1 & D\left(p 1, p_{2}\right) & 0 & D\left(p_{2}, p_{3}\right) & D\left(p_{2}, p_{4}\right) \\
1 & D\left(p_{1}, p_{3}\right) & D\left(p_{2}, p_{3}\right) & 0 & D\left(p_{3}, p_{4}\right) \\
1 & D\left(p_{1}, p_{4}\right) & D\left(p_{2}, p_{4}\right) & D\left(p_{3}, p_{4}\right) & 0
\end{array}\right|
$$

with $p_{4}$ is the unknown location of the drone, $D\left(p_{4}, p_{1}\right), D\left(p_{4}, p_{2}\right)$ and $D\left(p_{4}, p_{3}\right)$ are the distances $\widehat{d_{1}}, \widehat{d_{2}}$ and $\widehat{d_{3}}$ that are computed from the RSS for a given receiver height. It is then possible to calculate the unknown position of the receiver $\left(p_{4}\right)$ with respect to three known transmitter coordinates $\left(p_{1}, p_{2}, p_{3}\right)$ using [26]:

$$
p_{4}=p_{1}+k_{1} v_{1}+k_{2} v_{2} \pm k_{3}\left(v_{1} v_{2}\right)
$$

where $v_{1}=p_{2}-p_{1}$ and $v_{2}=p_{3}-p_{1}$, and

$$
k_{1}=-\frac{D\left(p_{1}, p_{2}, p_{3} ; p_{1}, p_{3}, p_{4}\right)}{D\left(p_{1}, p_{2}, p_{3}\right)}, k_{2}=\frac{D\left(p_{1}, p_{2}, p_{3} ; p_{1}, p_{2}, p_{4}\right)}{D\left(p_{1}, p_{2}, p_{3}\right)}, k_{3}=\frac{\sqrt{D\left(p_{1}, p_{2}, p_{3}, p_{4}\right)}}{D\left(p_{1}, p_{2}, p_{3}\right)}
$$

The CMD algorithm then outputs $(\widehat{x}, \widehat{y}, \widehat{z})$ for each of the generated possible heights $\Delta h$, and then the cost function is used to estimate the receiver's height, $h$, and its corresponding location. 


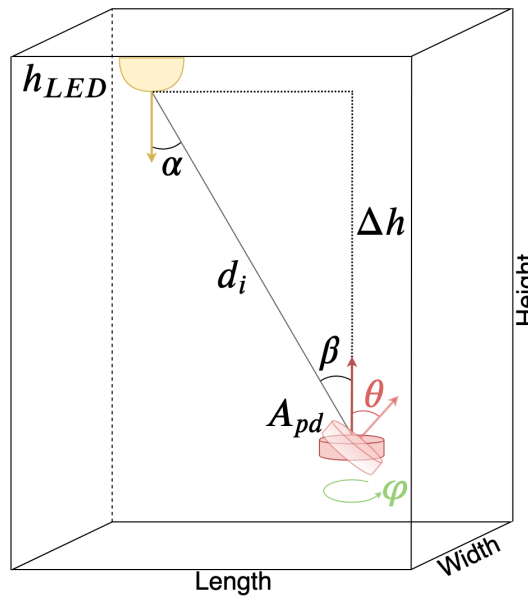

(a)

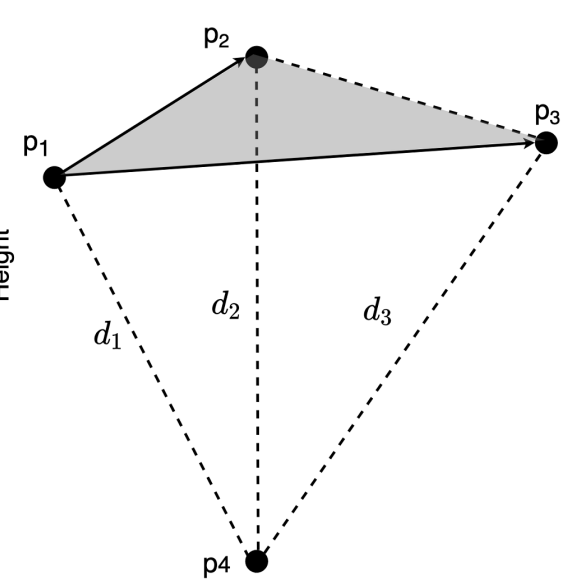

(b)

Figure 4: (a) The VLC channel parameters; (b) The parameters of the CMD trilateration algorithm.

\subsubsection{Linear Least Squares}

The LLS algorithm is used in this paper as a benchmark for comparison with the CMD algorithm as it is the most widely adopted trilateration positioning algorithm in VLP systems [27-29].

As the correct distances cannot be estimated directly without knowing the receiver's height, 2D trilateration using LLS is performed for each of the generated heights, $\Delta h$. The horizontal distance between the $\mathrm{LED}_{i}$ and the receiver is given by:

$$
d_{i}^{2}(\Delta h)=\left(x_{i}-x\right)^{2}+\left(y_{i}-y\right)^{2}=x^{2}-2 x x_{i}+x_{i}^{2}+y^{2}-2 y y_{i}+y_{i}^{2}
$$

These equations can be expressed in a matrix form as $\mathrm{b}=\mathrm{Ax}$, where

$$
b=\frac{1}{2}\left[\begin{array}{c}
d_{1}^{2}(\Delta h)-x_{1}^{2}-y_{1}^{2}-d_{N}^{2}(\Delta h)+x_{N}^{2}+y_{N}^{2} \\
d_{2}^{2}(\Delta h)-x_{2}^{2}-y_{2}^{2}-d_{N}^{2}(\Delta h)+x_{N}^{2}+y_{N}^{2} \\
\vdots \\
d_{N-1}^{2}(\Delta h)-x_{N-1}^{2}-y_{N-1}^{2}-d_{N}^{2}(\Delta h)+x_{N}^{2}+y_{N}^{2}
\end{array}\right]
$$




$$
A=\left[\begin{array}{cc}
x_{1}-x_{N} & y_{1}-y_{N} \\
x_{2}-x_{N} & y_{2}-y_{N} \\
\vdots & \vdots \\
x_{N-1}-x_{N} & y_{N-1}-y_{N}
\end{array}\right], x=\left[\begin{array}{l}
x \\
y
\end{array}\right]
$$

The algorithm then outputs the estimated position $\left[\begin{array}{l}\widehat{x} \\ \widehat{y}\end{array}\right]$ for each of the generated possible heights $(\Delta h)$ using:

$$
x=\left(A^{T} A\right)^{-1} A^{T} b
$$

\subsubsection{Cost function}

Once all of the possible receiver locations have been generated using (6) and (10) for both algorithms, the final most probable 3D position of the receiver is found at the minimum of the cost function $C(h)$ as [19]:

$$
C(h)=\frac{1}{N} \sum_{i=1}^{N}\left[\widehat{d}_{i}(h)-\sqrt{\left(\widehat{x}(h)-x_{i}\right)^{2}+\left(\widehat{y}(h)-y_{i}\right)^{2}+\left(\widehat{z}(h)-z_{i}\right)^{2}}\right]^{2}
$$

where $C(h)$ is the average squared error between the estimated distances $\widehat{d}_{i}$ using (2), and the distances of the estimated 3D location of the unknown receiver calculated using (6) and (10). It should be noted that the cost function minimization described above can be used in conjunction with any 2 D trilateration algorithm [24].

The positioning error, which is the distance difference between the final calculated position and the actual position of the receiver, is calculated using:

$$
D_{\text {error }}=\sqrt{(\widehat{x}-x)^{2}+(\widehat{y}-y)^{2}+(\widehat{z}-z)^{2}}
$$

where $z=h$. The CMD algorithm only requires three signals to estimate the receiver's position while the LLS generally utilizes all the received signals. In our experiment, the LLS algorithm in (8) is restricted to use only the strongest three signals to ensure a fair comparison. Also, restricting the LLS to use only the strongest signals has been shown to increase the positioning accuracy and lessen the impact of multipath reflection $[30,31]$. The cost function on the other hand uses all four signals from the LEDs for the minimization, as three LEDs do not suffice for an unambiguous 3D localization. 


\section{Results}

The performance of the algorithms is experimentally evaluated for different parameters in terms of positioning error while considering different realistic factors: (i) different LED configurations, (ii) different receiver tilt angles, and (iii) introduced multipath reflection through the inclusion of a storage rack. Moreover, the results section also examines the performance of the algorithms for a $2 \mathrm{D}$ system. In this case, the height of the receiver is assumed to be exactly known through the use of an additional sensor.

\subsection{Positioning Accuracy for Untilted Receiver}

\subsubsection{Square Configuration}

Figure 5 (a) shows the CDF using the CMD and LLS algorithms for a $2 \mathrm{D}$ and $3 \mathrm{D}$ positioning system. The median $\left(p_{50}\right)$ and maximal $\left(p_{90}\right) 2 \mathrm{D}$ errors recorded using the LLS algorithm are $11.7 \mathrm{~cm}$ and $26.7 \mathrm{~cm}$, while these are $9.9 \mathrm{~cm}$ and $15.8 \mathrm{~cm}$ using the CMD algorithm. In a 3D system, the measured median error is $17.1 \mathrm{~cm}$ and the maximal error is $88.4 \mathrm{~cm}$ for the LLS algorithm while the CMD algorithm achieves a median error of $55.9 \mathrm{~cm}$ and a maximal error of $177.9 \mathrm{~cm}$. The positioning errors for the 2D estimation are much smaller than the $3 \mathrm{D}$ estimation. This is due to the height being known to the receiver, avoiding the need for the cost function and eliminating the 3D positioning ambiguity [19]. In the case of 2D positioning, the CMD outperforms the LLS algorithms slightly while the LLS algorithm outperforms the CMD algorithm in a 3D system. However, the 3D estimation for both algorithms is unreliable due to the high positioning errors under the square configuration. This is caused by the position ambiguity in a square configuration as expected and further analyzed in our previous work [19, 24]. The issue arises because some locations in the room have the same received power values, and distances once converted, as other locations, which occurs due to the radiation pattern's geometrical properties [32].

\subsubsection{Star Configuration}

Figure 5 (b) shows the CDF of the positioning errors using the star arrangement of LEDs for both $2 \mathrm{D}$ and 3D position estimation. The overall error values have decreased noticeably when compared with the square arrangement as the position ambiguity is not present in the star configuration. The performance of the LLS and CMD algorithms are very similar for the 


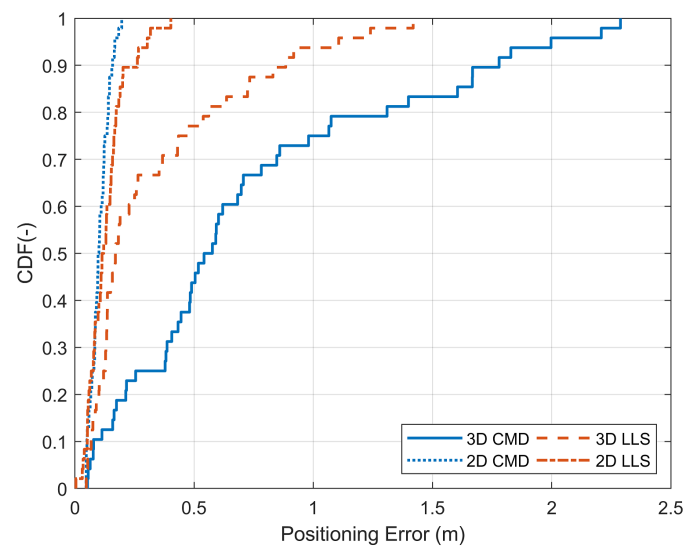

(a)

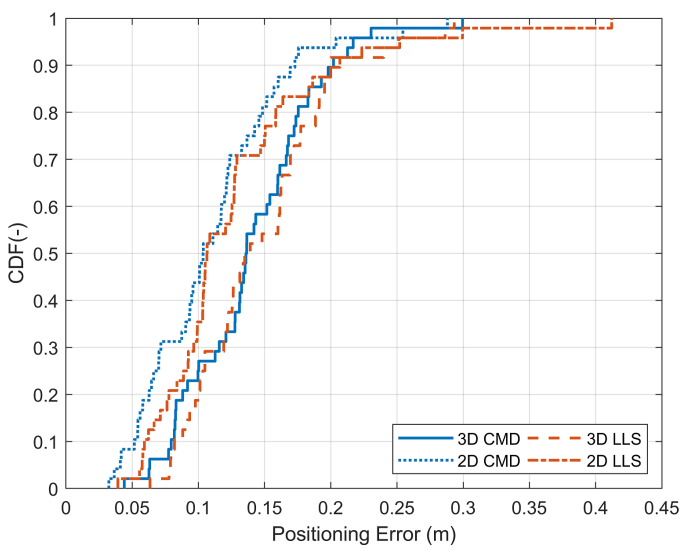

(b)

Figure 5: The CDF of the 2D and 3D positioning errors for both algorithms with a parallel receiver. (a) Under a square LED configuration; (b) Under a star LED configuration.

3D system with the median and maximal errors achieved using the LLS algorithm are $10.6 \mathrm{~cm} \& 24.9 \mathrm{~cm}$, and $10.5 \mathrm{~cm} \& 21.1 \mathrm{~cm}$ using the CMD algorithm, respectively. In the case of the $2 \mathrm{D}$ system, median and maximal positioning errors of 8 and $25.2 \mathrm{~cm}$ were measured using the LLS algorithm and $6.7 \mathrm{~cm} \& 14.6 \mathrm{~cm}$ using the CMD algorithm. Note that most of the large errors occurred at heights of more than 2 meters as can be seen in Figure 6, which depicts the estimated 3D paths and shows a deviation when the receiver is over 2 meters.

\subsection{Positioning Accuracy for a tilted receiver}

The errors introduced by the receiver tilt are due to the assumption in (2) that the transmitters' and receiver's plane are perfectly parallel to each other. This assumption is widely adopted due to its simplicity. However, it is unrealistic as it is almost impossible to achieve perfectly parallel planes in real-life settings, as even a $1^{\circ}$ difference can increase the positioning error [33]. This is especially important when considering the use of a VLP system with aerial receivers, as they tilt for movement. Therefore, the effect of tilting on the performance of positioning algorithms is investigated here.

To accurately assess the effect of the receiver's tilt, the receiver is mounted 


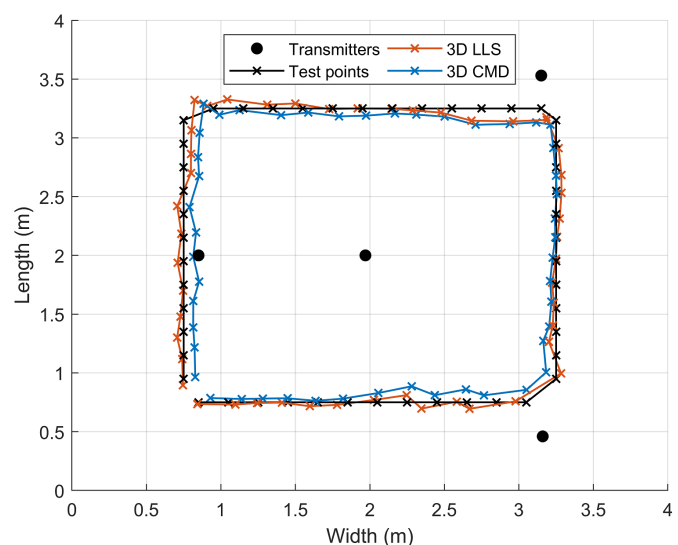

(a)

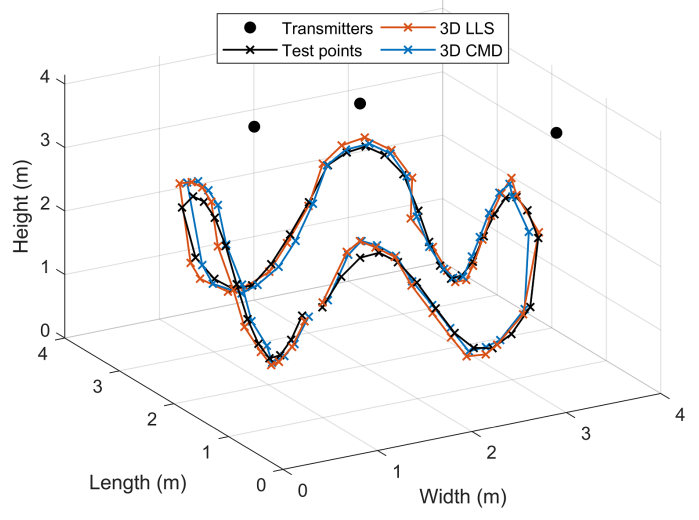

(b)

Figure 6: An illustration of the estimated paths under a star configuration when the receiver is parallel. (a) A top-view of the test points and the estimated 3D positions using the LLS and CMD algorithms; (b) a 3D view of the test points and the estimated points.

on a Thorlabs GNL10/ $\mathrm{M}^{2}$ goniometer with a range of $\pm 10^{\circ}$ and a precision of $1^{\circ}$ as shown in the inset of Figure $1(\mathrm{~b})$. Two tilt angles of $5^{\circ}$ and $10^{\circ}$ are considered and investigated. The tilt of the receiver is set to a forward tilt angle, meaning that the receiver is always facing the direction of movement along the path outlined earlier in Section 3 and shown in Figure 2 (a). The forward tilt is introduced here because UAVs normally tilt forward to move.

\subsubsection{Square Configuration}

Figure 7 (a) shows the CDF of the positioning errors using the squareshaped LED configuration for both 2D and 3D estimation with a receiver tilt angle $\theta=5^{\circ}$. The measured median and maximal errors using the LLS algorithm were $9.5 \mathrm{~cm}$ and $17.8 \mathrm{~cm}$, and it is $8.8 \mathrm{~cm}$ and $15.3 \mathrm{~cm}$ when the CMD algorithm is used. In a 3D system, the median and maximal errors for 2D using the LLS algorithm are 17.4 and $76.9 \mathrm{~cm}$, while it is 62.9 and $177.5 \mathrm{~cm}$ when the CMD algorithm is used. The results show that LLS outperforms the CMD algorithm in a square configuration. Figure 7 (c) shows the performance of the system with a receiver tilt of $10^{\circ}$. For a $2 \mathrm{D}$ system,

\footnotetext{
${ }^{2}$ https://www.thorlabs.com/thorproduct.cfm?partnumber=GNL10/M
} 
Table 3: A summary of the experimentally obtained median and maximal positioning errors for the two LED configurations for $2 \mathrm{D}$ and 3D localization when the receiver has a tilt of $0^{\circ}, 5^{\circ}$, and $10^{\circ}$.

\begin{tabular}{ccccccccc}
\hline Positioning Error $(\mathbf{c m})$ & 2D LLS & \multicolumn{2}{c}{ 2D CMD } & \multicolumn{2}{c}{ 3D LLS } & \multicolumn{2}{c}{ 3D CMD } \\
\hline & $\boldsymbol{p}_{\mathbf{5 0}}$ & $\boldsymbol{p}_{\mathbf{9 0}}$ & $\boldsymbol{p}_{\mathbf{5 0}}$ & $\boldsymbol{p}_{\mathbf{9 0}}$ & $\boldsymbol{p}_{\mathbf{5 0}}$ & $\boldsymbol{p}_{\mathbf{9 0}}$ & $\boldsymbol{p}_{\mathbf{5 0}}$ & $\boldsymbol{p}_{\mathbf{9 0}}$ \\
Square $\left(\theta=0^{\circ}\right)$ & 11.7 & 26.2 & 9.9 & 15.8 & 17.1 & 88.4 & 55.9 & 177.9 \\
Star $\left(\theta=0^{\circ}\right)$ & 8 & 25.2 & 6.7 & 14.6 & 10.6 & 24.9 & 10.5 & 21.1 \\
Square $\left(\theta=5^{\circ}\right)$ & 9.5 & 17.8 & 8.8 & 15.3 & 17.4 & 76.9 & 62.9 & 177.5 \\
Star $\left(\theta=5^{\circ}\right)$ & 10.7 & 20.7 & 10.4 & 17.3 & 13.7 & 20 & 13.6 & 20.2 \\
Square $\left(\theta=10^{\circ}\right)$ & 19.4 & 28.1 & 15.6 & 22.8 & 27.1 & 186.4 & 106.7 & 181.8 \\
Star $\left(\theta=10^{\circ}\right)$ & 23.2 & 36.3 & 18.8 & 31.3 & 22.7 & 32.2 & 21.6 & 34.2 \\
\hline
\end{tabular}

the recorded median errors are 19.4 and $15.6 \mathrm{~cm}$ for the LLS and CMD algorithms, respectively. The largest errors recorded are when a 3D system was used with a receiver tilt $\theta=10^{\circ}$ with a median of $27.1 \mathrm{~cm}$ using LLS, and $106.7 \mathrm{~cm}$ using CMD. These results again demonstrate the unreliability of using a square layout when implementing the algorithm. Table 3 lists a summary of the obtained accuracies across all tilt angles for the CMD and LLS algorithms under the two LED configurations.

\subsubsection{Star Configuration}

Figure 7 (b) shows the CDF of the positioning error for the entire path when the receiver is tilted by $\theta=5^{\circ}$ under a star configuration. When the LLS algorithm is used for 3D positioning, the median error is $13.7 \mathrm{~cm}$ and the maximal error is $20 \mathrm{~cm}$. In the case of $2 \mathrm{D}$ positioning, the median error is $10.7 \mathrm{~cm}$ and the maximal error is $20.7 \mathrm{~cm}$, which is slightly better than $3 \mathrm{D}$ positioning. When the CMD algorithm is used for $2 \mathrm{D}$ positioning, the median and maximal errors recorded were 10.4 and $17.3 \mathrm{~cm}$, and in the case of 3D positioning, the median and maximal errors are 13.6 and $20.2 \mathrm{~cm}$.

The measured positioning errors with $\theta=10^{\circ}$ are shown in Figure $7(\mathrm{~d})$. Median and maximal errors for the 2D system are $23.2 \mathrm{~cm}$ and $36.3 \mathrm{~cm}$ for the LLS algorithm, while it is $18.8 \mathrm{~cm}$ and $31.3 \mathrm{~cm}$ for the CMD algorithm, respectively. In a $3 \mathrm{D}$ positioning system, the median and maximal errors were $22.7 \mathrm{~cm}$ and $32.2 \mathrm{~cm}$ when using the LLS algorithm, and $21.6 \mathrm{~cm}$ and $34.2 \mathrm{~cm}$ using the CMD algorithm.

In can be noticed that some of the errors are higher under a square setting with an untilted receiver than when the receiver is $\theta=5^{\circ}$, see Table 3 . The increase is due to some of the measured samples having large errors that have skewed the maximal errors. Note that, the tilt effect could be alleviated 


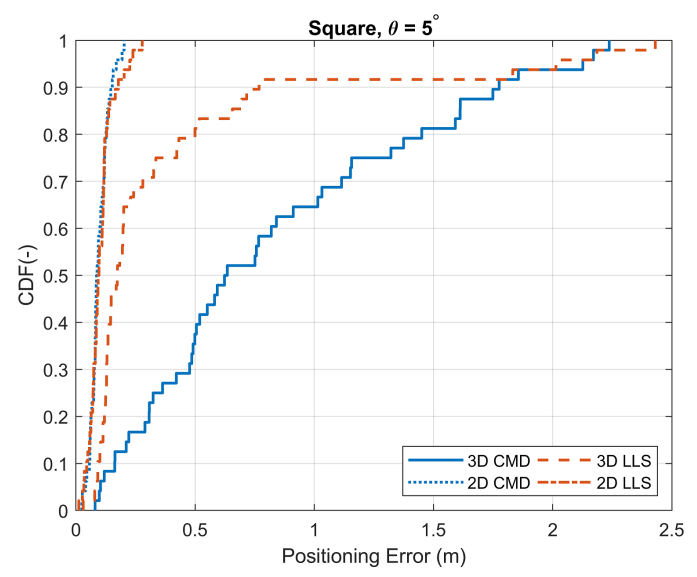

(a)

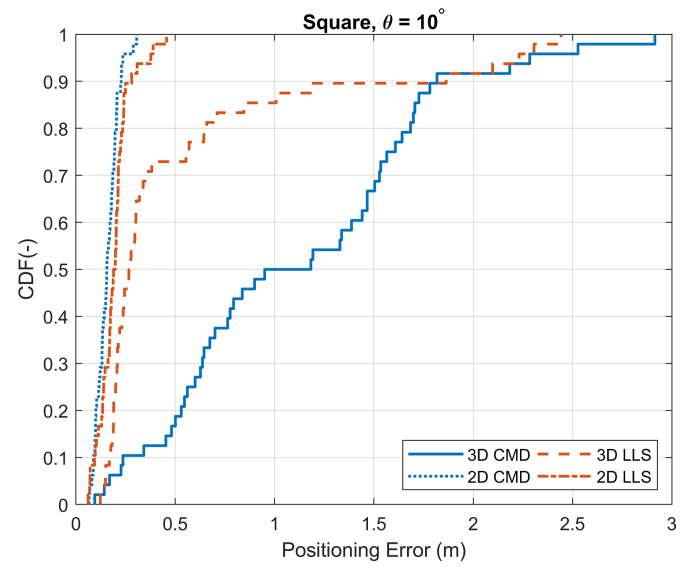

(c)

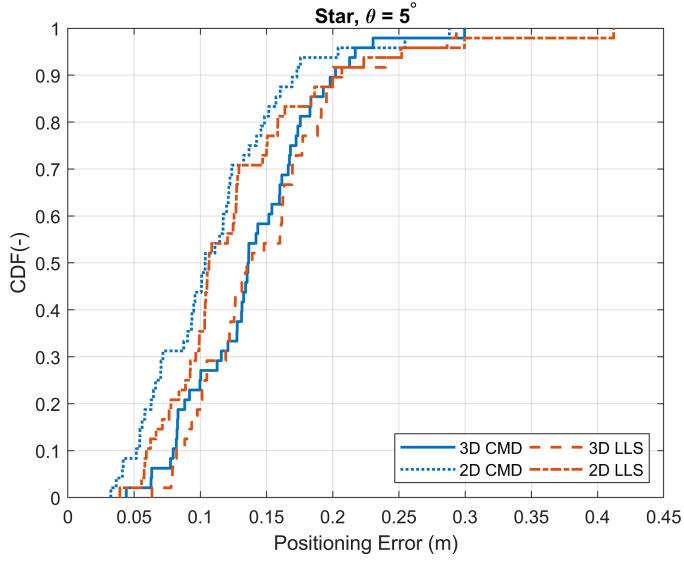

(b)

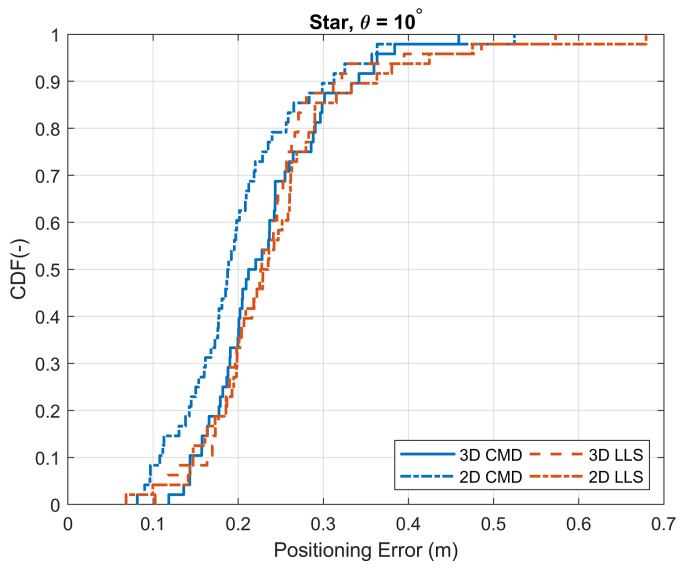

(d)

Figure 7: The CDF of the 2D and 3D positioning errors for both algorithms with receiver tilt, $\theta$. (a) Square LED configuration with a receiver tilt of $5^{\circ}$; (b) Star LED configuration with a receiver tilt of $5^{\circ}$; (c) Square LED configuration with a receiver tilt of $10^{\circ}$; (d) Star LED configuration with a receiver tilt of $10^{\circ}$. 


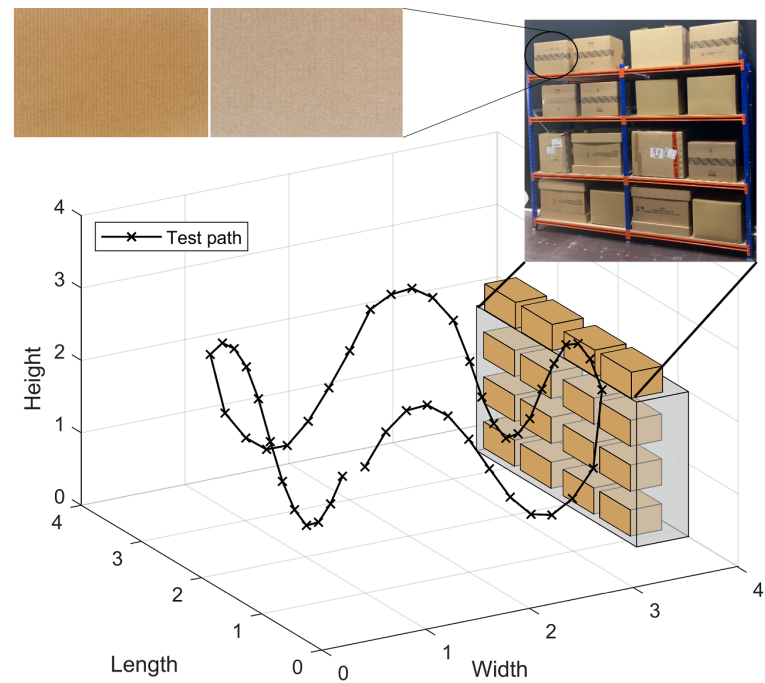

(a)

Figure 8: A 3D view of the storage rack and test path in relation to the room. The inset shows the storage rack stocked with boxes with reflectivity of $33 \%$ and $42 \%$ depending on color tone.

through compensating its value, which can be performed by receivers that are equipped with an IMU/gyroscope $[34,35]$ or with algorithms such as simultaneous positioning and orientating (SPAO) [36].

\subsection{Positioning Accuracy in the Presence of Multipath Reflections}

Industrial environments are one of the areas where an indoor positioning system could prove valuable. As discussed previously, UAVs and AGVs can be deployed in warehouses and storage facilities with the help of VLP systems for inventory management applications. In order to replicate an industrial warehouse, a metal storage rack was added to the room as shown in Figure 2. The rack is placed at one side of the room along the path and is stocked with different-sized boxes as shown in the inset of Figure 8. The height of the storage rack is $2 \mathrm{~m}$ and measures $2.36 \mathrm{~m}$ when stocked with boxes and has a length of $2.66 \mathrm{~m}$. The storage rack is placed $26 \mathrm{~cm}$ away from the path test points that runs parallel to it. A 3D illustration of the storage rack and the test points in the room can be seen in Figure 8.

Research work has shown that reflections degrade the performance of VLP systems, especially when near highly reflective surfaces such as white painted walls that have a reflectivity of around $70 \%$ [30]. In our case, the 


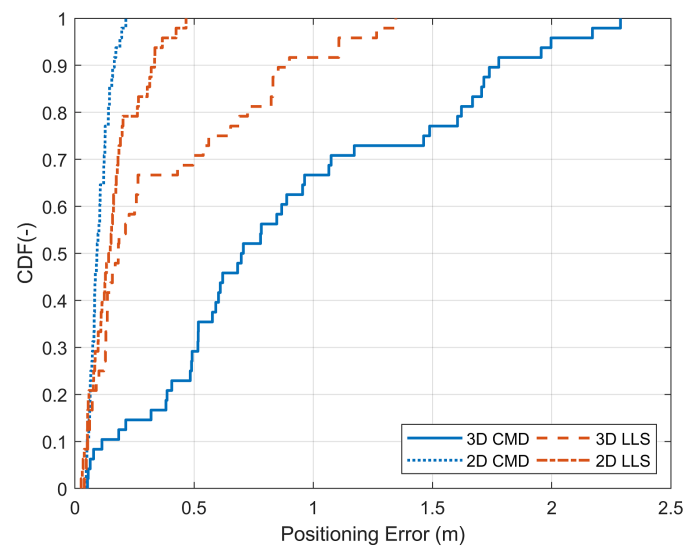

(a)

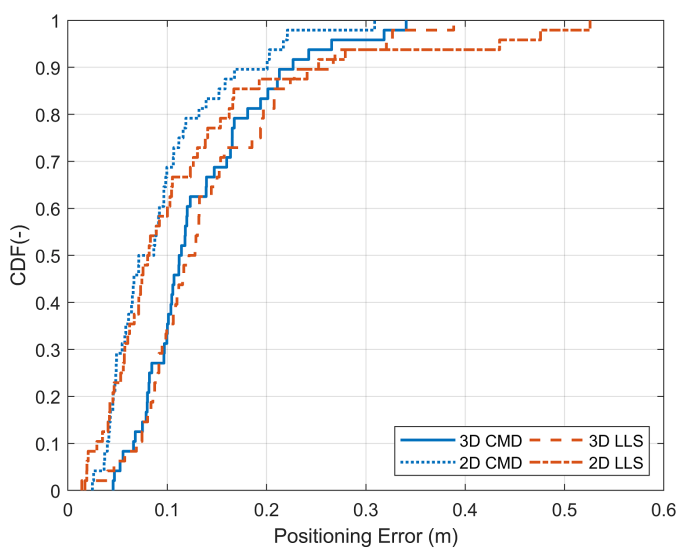

(b)

Figure 9: The CDF of the 2D and 3D positioning errors for both algorithms with a parallel receiver in the presence of a storage rack. (a) Under a square LED configuration; (b) and under a star LED configuration.

reflectivity of the boxes ranges between $33-42 \%$ depending on the color tone of the cardboard as demonstrated in the inset of Figure 8. These values were obtained using DIALux ${ }^{3}$. The same measurement procedure and scenarios outlined earlier(two LED configurations with 2D and 3D using the CMD and LLS trilatertaion algorithms) have been repeated, and then the positioning error was calculated using (12).

\subsubsection{Untilted Receiver}

Figure 9 (a) shows the CDF of the positioning errors using a square configuration with the inclusion of the storage rack. In the $2 \mathrm{D}$ system, the median and maximal errors using the LLS algorithm are $14.5 \mathrm{~cm}$ and $33.4 \mathrm{~cm}$, whereas the CMD algorithm achieve a median and maximal value of $9.3 \mathrm{~cm}$ and $16.5 \mathrm{~cm}$ using the CMD algorithm.

Figure 9 (b) shows the CDF of the positioning errors using the LLS and CMD algorithms under a star LED configuration. The median and maximal 2D errors using the LLS algorithm are $8.1 \mathrm{~cm}$ and $25.2 \mathrm{~cm}$, whereas a median error of $7.9 \mathrm{~cm}$ and a maximal error of $20.1 \mathrm{~cm}$ when the CMD algorithm is

\footnotetext{
${ }^{3}$ https://www.dial.de/en/dialux/
} 


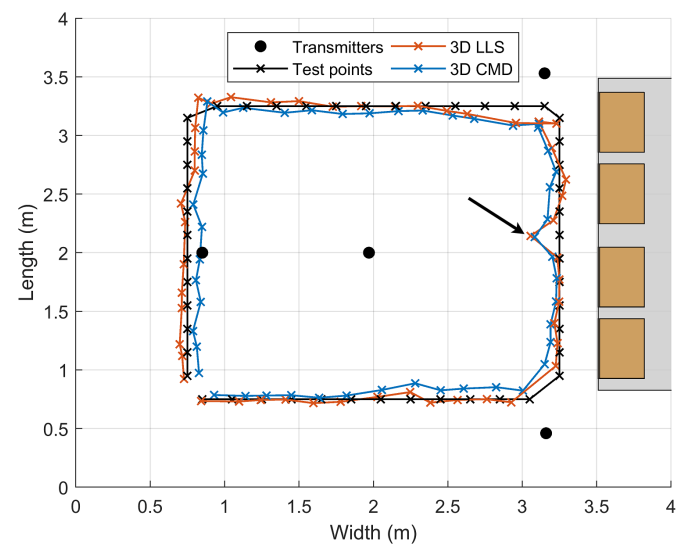

(a)

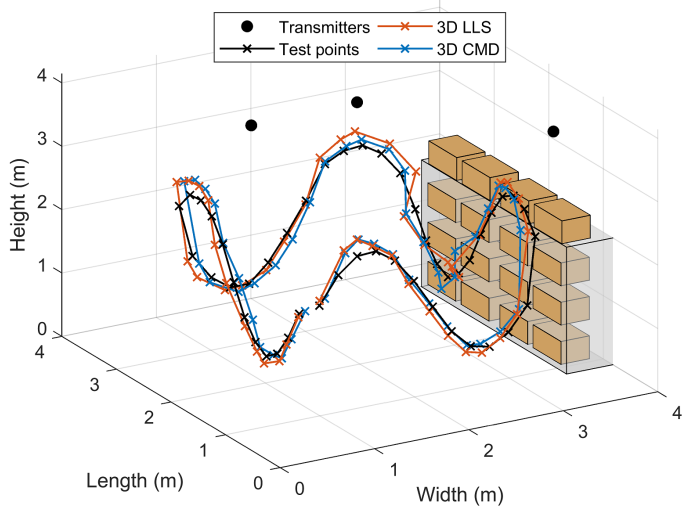

(b)

Figure 10: (a) A top-view of the test points and the estimated 3D positions using the LLS and CMD algorithms when the receiver is parallel; (b) a 3D view of the test points and the estimated $3 \mathrm{D}$ points.

used. The errors increase slightly in a 3D system with median and maximal errors of $12.5 \mathrm{~cm}$ and $26.7 \mathrm{~cm}$ using the LLS algorithm. In a 3D system, the CMD algorithm achieved a median and a maximal value of $11.3 \mathrm{~cm}$ and $22.7 \mathrm{~cm}$.

Figure 10 illustrates the estimated paths using the CMD and LLS algorithms. The errors on the right side and top-right side near the storage rack are due to reflections from the boxes and the metal rods [37]. The bottomright path is not particularly affected as some receiver heights are higher than the storage rack. Figure 10 (a) demonstrates the detrimental impact of reflections for the points that run parallel to the storage rack. One particular point directly across the metal rod is heavily affected by the multipath reflection emanating from the central LED and as highlighted in Figure 10 (a). The positioning error for that point in the 3D systems reported an error of $19.7 \mathrm{~cm}$ using the LLS algorithm, increasing from $6.7 \mathrm{~cm}$ when the point was calculated prior to adding a storage rack. Using the CMD algorithm, that specific point reported an error of $26.6 \mathrm{~cm}$, whereas it was $8.6 \mathrm{~cm}$ prior to the addition of the storage rack. Overall, the results do not differ greatly when compared with the results in the absence of the storage rack except for the points that are nearest to storage rack. 


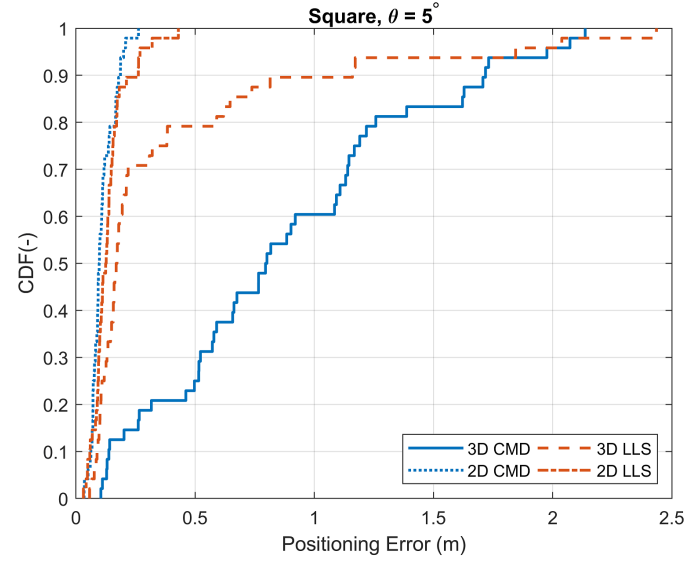

(a)

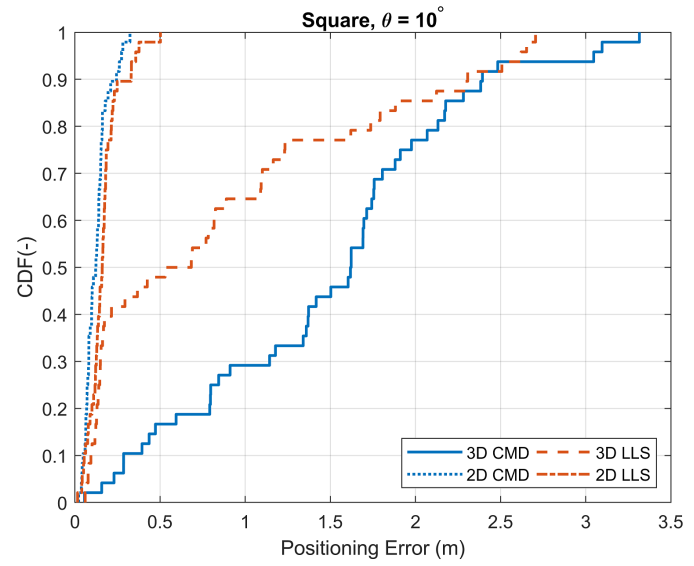

(c)

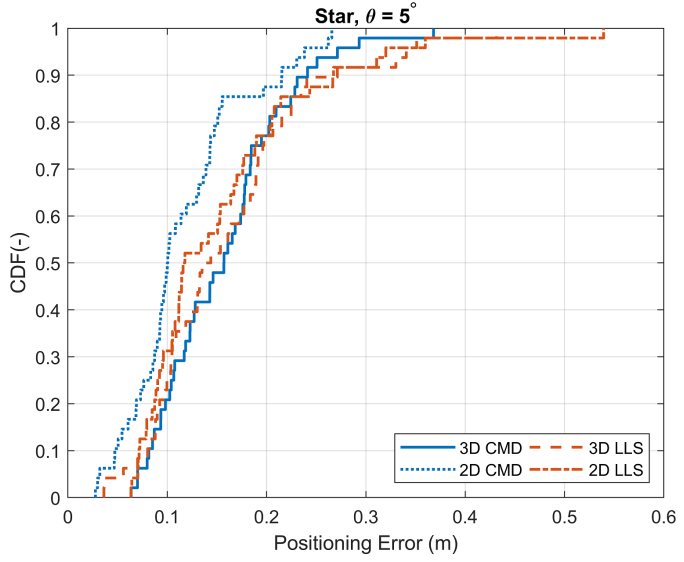

(b)

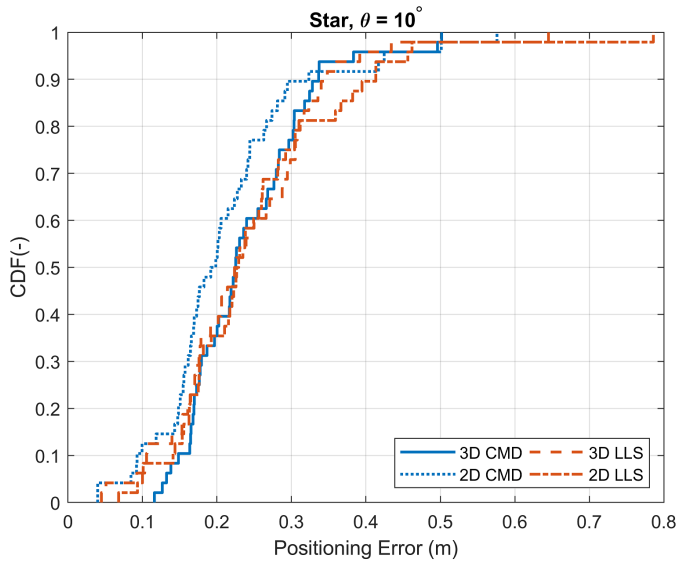

(d)

Figure 11: The CDF of the 2D and 3D positioning errors for both algorithms when the receiver is tilted and with the inclusion of a storage rack. (a) Square configuration with a receiver tilt of $5^{\circ}$; (b) Star configuration with a receiver tilt of $5^{\circ}$; (c) Square configuration with a tilt of $10^{\circ}$; (d) Star configuration with a receiver tilted $10^{\circ}$. 
Table 4: A summary of the experimentally obtained median and maximal positioning errors for the two LED configurations for $2 \mathrm{D}$ and 3D localization when the receiver has a tilt of $0^{\circ}, 5^{\circ}$, and $10^{\circ}$ in the presence of a storage rack.

\begin{tabular}{ccccccccc}
\hline Positioning Error $\mathbf{( c m})$ & 2D LLS & \multicolumn{2}{c}{ 2D CMD } & 3D LLS & \multicolumn{2}{c}{ 3D CMD } \\
\hline & $\boldsymbol{p}_{\mathbf{5 0}}$ & $\boldsymbol{p}_{\mathbf{9 0}}$ & $\boldsymbol{p}_{\mathbf{5 0}}$ & $\boldsymbol{p}_{\mathbf{9 0}}$ & $\boldsymbol{p}_{\mathbf{5 0}}$ & $\boldsymbol{p}_{\mathbf{9 0}}$ & $\boldsymbol{p}_{\mathbf{5 0}}$ & $\boldsymbol{p}_{\mathbf{9 0}}$ \\
Square $\left(\theta=0^{\circ}\right)$ & 14.5 & 33.4 & 9.3 & 16.5 & 18.2 & 90 & 70.2 & 177.8 \\
Star $\left(\theta=0^{\circ}\right)$ & 8.1 & 25.2 & 7.9 & 20.1 & 12.2 & 26.7 & 11.3 & 22.7 \\
Square $\left(\theta=5^{\circ}\right)$ & 12.6 & 26.2 & 9.8 & 18.7 & 17.1 & 116 & 79.8 & 171.8 \\
Star $\left(\theta=5^{\circ}\right)$ & 11.7 & 26.7 & 10 & 21.5 & 13.9 & 27.1 & 15.7 & 24.1 \\
Square $\left(\theta=10^{\circ}\right)$ & 16 & 33 & 12.3 & 24.4 & 60.7 & 230.7 & 162 & 239.3 \\
Star $\left(\theta=10^{\circ}\right)$ & 22.8 & 41.3 & 19.5 & 32.3 & 22.5 & 34.8 & 22.5 & 33.7 \\
\hline
\end{tabular}

\subsubsection{Tilted Receiver}

Similar to Subsection 5.2, the measurements are repeated with the receiver tilted by $5^{\circ}$ and $10^{\circ}$. This means that the system/receiver will suffer from both the effects of tilt and multipath reflections. Figure 11 shows the $\mathrm{CDF}$ of the positioning errors when the receiver is tilted $5^{\circ}$ and $10^{\circ}$ for both LED configurations. Under a square setting and when the receiver is tilted by $5^{\circ}$, the measured median and maximal $2 \mathrm{D}$ errors using the LLS algorithm were 12.6 and $26 \mathrm{~cm}$, whereas it is $9.8 \mathrm{~cm}$ and $18.7 \mathrm{~cm}$ when the CMD algorithm is used, see Figure 11 (a). In the 3D system, the measured median and maximal values are $17.1 \mathrm{~cm}$ and $116 \mathrm{~cm}$ using the LLS algorithm. Using the CMD algorithm achieved 3D median and maximal values of 79.8 and $171.8 \mathrm{~cm}$. Here, the results show that $70 \%$ of the errors in a 3D system using the LLS algorithm are below $22 \mathrm{~cm}$, as shown in Figure 11 (a).

In the $2 \mathrm{D}$ system when the receiver is tilted by $10^{\circ}$, the LLS algorithm achieved median and maximal errors of 16 and $33 \mathrm{~cm}$. While the CMD algorithm achieved median and maximal values of 12.3 and $24.4 \mathrm{~cm}$. In the 3D system, the LLS algorithm reported a median of $60.7 \mathrm{~cm}$ and using the CMD algorithm reported $1.62 \mathrm{~m}$ as shown in Figure 11 (c). As expected, the errors increase when the tilt is increased to $10^{\circ}$.

Figure 11 (b) demonstrates the CDF for a receiver with a tilt of $5^{\circ}$ under the star arrangement. Using the LLS algorithm, the achieved 2D median and maximal errors are $11.7 \mathrm{~cm}$ and $26.7 \mathrm{~cm}$, whereas they are $10 \mathrm{~cm}$ and $21.5 \mathrm{~cm}$ when the CMD algorithm is used. For the 3D positioning system, the median error using the LLS algorithm is $13.9 \mathrm{~cm}$, an increase of $13.9 \%$ when compared with an untilted receiver. Using the CMD algorithm, the median is $15.7 \mathrm{~cm}$, increasing by $39 \%$ to when the receiver was untilted. When 


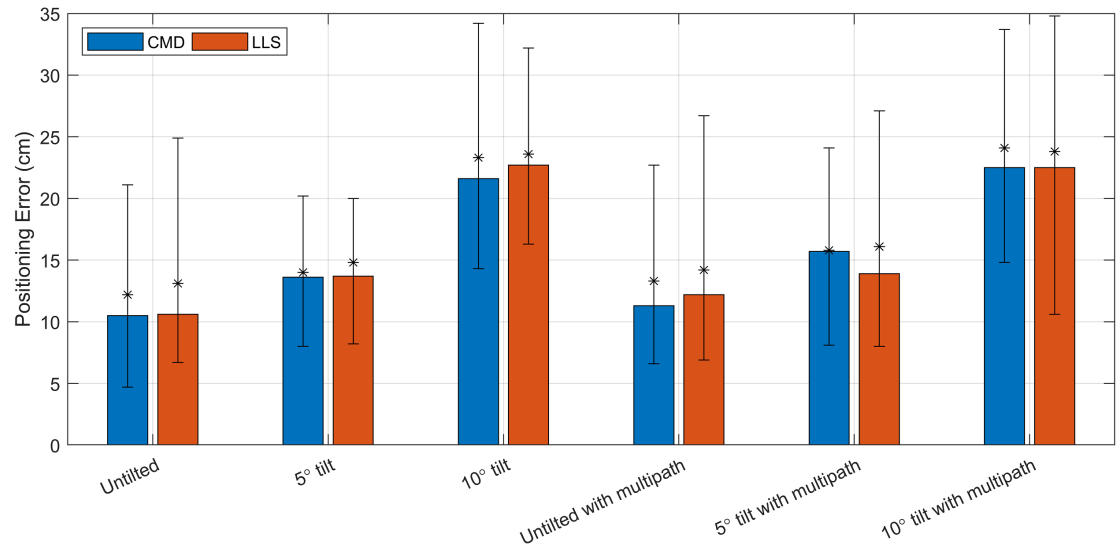

Figure 12: The bars show the achieved 3D median errors using the CMD and LLS trilateration algorithms under a star configuration, the error bars show the $10 \%$ and $90 \%$ quantiles, and the asterisks represent the mean error.

the tilt is $5^{\circ}$, the CMD algorithm outperforms the LLS algorithm when it comes to $2 \mathrm{D}$ positioning. The results, however, are nearly identical in the 3D positioning system.

When the receiver's tilt is set to $10^{\circ}$ under a star arrangement, the performance of the two algorithms in both 2D and 3D positioning system are similar. The median 3D error reported $22.5 \mathrm{~cm}$ for both algorithms, see Figure 11. Table 4 lists a summary of the obtained accuracies across all tilt angles in the presence of the storage rack. Compared to when the receiver was untilted, the errors increased by $84 \%$ using the LLS algorithm and doubled when using the CMD algorithm.

\section{Discussion}

We experimentally evaluated and compared two different VLP trilateration algorithms in a $4 \mathrm{~m} \times 4 \mathrm{~m} \times 4.1 \mathrm{~m}$ room under two different LED configurations for both $2 \mathrm{D}$ and $3 \mathrm{D}$ systems. The performances of the algorithms were also examined in the presence of a storage rack to examine the effects of multipath reflections. Our experiments demonstrated the impracticality of using a square-shaped configuration and showed the higher positioning accuracy of a star-shaped configuration. Previous simulation work identified an issue when the LEDs were placed in a square configuration [19]. 
Therefore, a star-shaped configuration was proposed. This shortcoming was experimentally examined in this paper.

The results under a star configuration were highly more accurate compared to the square configuration. The 3D median error achieved using LLS and CMD were $10.6 \mathrm{~cm}$ and $10.5 \mathrm{~cm}$, respectively. When a tilt of $5^{\circ}$ was introduced, the 3D median errors increased slightly to $13.7 \mathrm{~cm}$ and $13.6 \mathrm{~cm}$ for LLS and CMD, an increase of $29.3 \%$ and $29.5 \%$. A tilt of $10^{\circ}$ increased the 3D median errors of LLS and CMD to $22.7 \mathrm{~cm}$ and $21.6 \mathrm{~cm}$, corresponding to an increase of $114.2 \%$ and $106 \%$ when compared with a horizontal receiver. From these results, we can conclude that the positioning error increases by around $30 \%$ if the receiver is tilted by $5^{\circ}$, and essentially doubles when the receiver is tilted by $10^{\circ}$. Figure 12 shows the median errors for all of the considered scenarios under a star arrangement, the error bars show the $10 \%$ and $90 \%$ quantiles, and the asterisks show the mean error. A slight difference in terms of positioning error between the median and mean can be seen for some of the scenarios.

The effect of multipath reflections on the performance of VLP systems was also examined. A metallic storage rack filled with boxes was added in the evaluated room and tested with a horizontal receiver with a receiver tilt of $5^{\circ}$ and $10^{\circ}$. The results for a $3 \mathrm{D}$ system under a star configuration reported a median error of $12.2 \mathrm{~cm}$ using LLS, an increase of $15 \%$ when compared with an empty room. Using the CMD algorithm, the median error was $11.3 \mathrm{~cm}$, which represents an increase of $7.6 \%$ compared to its performance in an empty room. The storage rack was $26 \mathrm{~cm}$ away from the closest points and the impact of reflections on one particular point (pointed out in Figure 10) increased the positioning error in a 3D system using the LLS algorithm by $13 \mathrm{~cm}$, and by $18 \mathrm{~cm}$ using the CMD algorithm [37]. This points out the severity of multipath reflections from metallic structures. As mentioned before, both algorithms in this paper select the three strongest signals to increase the positioning accuracy and lessen the impact of multipath reflections as noted in $[30,31]$. However, while the impact of reflections may have been reduced, it is still not sufficient enough in limiting the degrading effect of reflections.

The differences in the performances of the algorithms are because they differ mathematically in how they calculate the receiver's position. The CMD method is an analytic procedure, that calculates a point through geometric interrelations [38]. Whereas the least square method is a numeric procedure that calculates the point at which the distance from three circles intersects. 
The observation that the CMD trilateration algorithm outperforms the least square quadratic method has also been noted by researchers in [39] when they compared different trilateration algorithms.

It should be noted that some of the errors observed in the experiments could also be caused by other factors. The experimentally adjusted tilt angle can be slightly different from the intended values, the LED having small unknown tilt angles [33], the LED radiation pattern not being perfectly Lambertian, and imperfections in the demultiplexing process.

\section{Conclusion}

In this paper, two VLP algorithms were experimentally analyzed and compared. The LLS and CMD algorithms were tested in a $4 \mathrm{~m} \times 4 \mathrm{~m} \times 4.1 \mathrm{~m}$ room with four LEDs. Two different LED configurations were compared using two different trilateration algorithms and their 2D and 3D performances were evaluated. The LLS and CMD algorithms achieved an accuracy of 10.6 and $10.5 \mathrm{~cm}$ in a 3D system, respectively. The performances of the algorithms were also examined in the presence of a storage rack to examine the effects of multipath reflections. We also experimentally demonstrated the impracticality of using a square-shaped configuration and showed the higher positioning accuracy of a star-shaped configuration. The proposed algorithm is suitable for real-time implementation based on our previous work reporting a computation time of $17 \mathrm{~ms}$, which can be further reduced to less than $2 \mathrm{~ms}$ using a fast search algorithm [19].

The presented work highlights the need to take into account the light arrangements to optimize the performance of a 3D VLP system as well as the effect of receiver tilt and multipath reflections on the performance of VLP systems. It also extended the use of a trilateration algorithm that is not wildly used into VLP systems. Future work could examine integrating an IMU sensor to compensate for the undesirable effects of tilt. Additional plans could also investigate the performance of the algorithm under a circular LED arrangement as the work in [40] reported that a circular arrangement offers a slightly higher illuminance uniformity than the optimum condition using rectangular LED arrangement.

\section{Acknowledgments}

The authors would like to thank Willem Raes and Nobby Stevens of DRAMCO, ESAT, KU LEUVEN, Belgium for providing the LED modules 
used in this work.

\section{Funding Information}

This research received no external funding.

\section{References}

[1] R. Xu, W. Chen, Y. Xu, S. Ji, A new indoor positioning system architecture using GPS signals, Sensors 15 (2015) 10074-10087. doi:10.3390/s150510074.

[2] F. Zafari, A. Gkelias, K. K. Leung, A survey of indoor localization systems and technologies, IEEE Communications Surveys Tutorials 21 (2019) 2568-2599. doi:10.1109/COMST.2019.2911558.

[3] H. Burchardt, N. Serafimovski, D. Tsonev, S. Videv, H. Haas, Vlc: Beyond point-to-point communication, IEEE Communications Magazine 52 (2014) 98-105.

[4] X. Guo, S. Shao, N. Ansari, A. Khreishah, Indoor localization using visible light via fusion of multiple classifiers, IEEE Photonics Journal 9 (2017) 1-16. doi:10.1109/JPHOT.2017.2767576.

[5] Y. Cai, W. Guan, Y. Wu, C. Xie, Y. Chen, L. Fang, Indoor high precision three-dimensional positioning system based on visible light communication using particle swarm optimization, IEEE Photonics Journal 9 (2017) 1-20. doi:10.1109/JPHOT.2017.2771828.

[6] C. Hsu, S. Liu, F. Lu, C. Chow, C. Yeh, G. Chang, Accurate indoor visible light positioning system utilizing machine learning technique with height tolerance, in: 2018 Optical Fiber Communications Conference and Exposition (OFC), 2018, pp. 1-3.

[7] Z. Xie, W. Guan, J. Zheng, X. Zhang, S. Chen, B. Chen, A highprecision, real-time, and robust indoor visible light positioning method based on mean shift algorithm and unscented Kalman filter, Sensors 19 (2019). doi:10.3390/s19051094. 
[8] H. Zhang, J. Cui, L. Feng, A. Yang, H. Lv, B. Lin, H. Huang, Highprecision indoor visible light positioning using deep neural network based on the Bayesian regularization with sparse training point, IEEE Photonics Journal 11 (2019) 1-10. doi:10.1109/JPHOT.2019.2912156.

[9] P. Du, S. Zhang, C. Chen, A. Alphones, W. Zhong, Demonstration of a low-complexity indoor visible light positioning system using an enhanced TDOA scheme, IEEE Photonics Journal 10 (2018) 1-10. doi:10.1109/JPHOT.2018.2841831.

[10] S. Zhang, W. Zhong, P. Du, C. Chen, Experimental demonstration of indoor sub-decimeter accuracy VLP system using differential PDOA, IEEE Photonics Technology Letters 30 (2018) 1703-1706. doi:10.1109/LPT.2018.2866402.

[11] Z. Li, L. Feng, A. Yang, Fusion based on visible light positioning and inertial navigation using extended Kalman filters, Sensors 17 (2017). doi:10.3390/s17051093.

[12] L. Li, P. Hu, C. Peng, G. Shen, F. Zhao, Epsilon: A visible light based positioning system, in: 11th USENIX Symposium on Networked Systems Design and Implementation (NSDI 14), USENIX Association, Seattle, WA, 2014, pp. 331-343.

[13] M. Yasir, S. Ho, B. N. Vellambi, Indoor positioning system using visible light and accelerometer, Journal of Lightwave Technology 32 (2014) 3306-3316. doi:10.1109/JLT.2014.2344772.

[14] S. Yang, H. Kim, Y. Son, S. Han, Three-dimensional visible light indoor localization using aoa and rss with multiple optical receivers, Journal of Lightwave Technology 32 (2014) 2480-2485. doi:10.1109/JLT.2014.2327623.

[15] W. Guan, S. Chen, S. Wen, Z. Tan, H. Song, W. Hou, High-accuracy robot indoor localization scheme based on robot operating system using visible light positioning, IEEE Photonics Journal 12 (2020) 1-16.

[16] W. Guan, X. Zhang, Y. Wu, Z. Xie, J. Li, J. Zheng, High precision indoor visible light positioning algorithm based on double leds using cmos image sensor, Applied Sciences 9 (2019). doi:10.3390/app9061238. 
[17] Y. Almadani, M. Ijaz, W. Joseph, S. Bastiaens, S. Rajbhandari, B. Adebisi, D. Plets, A novel 3d visible light positioning method using received signal strength for industrial applications, Electronics 8 (2019). doi:10.3390/electronics8111311.

[18] S. De Lausnay, L. De Strycker, J. Goemaere, N. Stevens, B. Nauwelaers, A visible light positioning system using frequency division multiple access with square waves, in: 2015 9th International Conference on Signal Processing and Communication Systems (ICSPCS), 2015, pp. 1-7. doi:10.1109/ICSPCS.2015.7391787.

[19] D. Plets, Y. Almadani, S. Bastiaens, M. Ijaz, L. Martens, W. Joseph, Efficient 3d trilateration algorithm for visible light positioning, Journal of Optics 21 (2019) 05LT01. doi:10.1088/2040-8986/ab1389.

[20] J. M. Kahn, J. R. Barry, Wireless infrared communications, Proceedings of the IEEE 85 (1997) 265-298. doi:10.1109/5.554222.

[21] Y. Almadani, M. Ijaz, S. Rajbhandari, U. Raza, B. Adebisi, Applications of visible light communication for distance estimation: a short survey, in: 2019 IEEE Jordan International Joint Conference on Electrical Engineering and Information Technology (JEEIT), 2019, pp. 261-265. doi:10.1109/JEEIT.2019.8717459.

[22] D. Plets, S. Bastiaens, L. Martens, W. Joseph, N. Stevens, On the impact of led power uncertainty on the accuracy of $2 \mathrm{~d}$ and $3 \mathrm{~d}$ visible light positioning, Optik 195 (2019) 163027. doi:10.1016/j.ijleo.2019.163027.

[23] D. Plets, S. Bastiaens, N. Stevens, L. Martens, W. Joseph, Monte-carlo simulation of the impact of led power uncertainty on visible light positioning accuracy, in: 2018 11th International Symposium on Communication Systems, Networks Digital Signal Processing (CSNDSP), 2018, pp. 1-6. doi:10.1109/CSNDSP.2018.8471838.

[24] D. Plets, S. Bastiaens, M. Ijaz, Y. Almadani, L. Martens, W. Raes, N. Stevens, W. Joseph, Three-dimensional visible light positioning: an experimental assessment of the importance of the leds' locations, in: 2019 International Conference on Indoor Positioning and Indoor Navigation (IPIN), 2019, pp. 1-6. doi:10.1109/IPIN.2019.8911763. 
[25] Z. Jia, C. Wu, Z. Li, Y. Zhang, B. Guan, The indoor localization and tracking estimation method of mobile targets in threedimensional wireless sensor networks, Sensors 15 (2015) 29661-29684. doi:10.3390/s151129661.

[26] F. Thomas, L. Ros, Revisiting trilateration for robot localization, IEEE Transactions on Robotics 21 (2005) 93-101. doi:10.1109/TRO.2004.833793.

[27] T.-H. Do, M. Yoo, An in-depth survey of visible light communication based positioning systems, Sensors 16 (2016). doi:10.3390/s16050678.

[28] Y. Zhuang, L. Hua, L. Qi, J. Yang, P. Cao, Y. Cao, Y. Wu, J. Thompson, H. Haas, A survey of positioning systems using visible led lights, IEEE Communications Surveys Tutorials 20 (2018) 1963-1988. doi:10.1109/COMST.2018.2806558.

[29] J. Luo, L. Fan, H. Li, Indoor positioning systems based on visible light communication: State of the art, IEEE Communications Surveys Tutorials 19 (2017) 2871-2893. doi:10.1109/COMST.2017.2743228.

[30] W. Gu, M. Aminikashani, P. Deng, M. Kavehrad, Impact of multipath reflections on the performance of indoor visible light positioning systems, Journal of Lightwave Technology 34 (2016) 2578-2587. doi:10.1109/JLT.2016.2541659.

[31] W. Tang, J. Zhang, B. Chen, Y. Liu, Y. Zuo, S. Liu, Y. Dai, Analysis of indoor VLC positioning system with multiple reflections, in: 2017 16th International Conference on Optical Communications and Networks (ICOCN), 2017, pp. 1-3. doi:10.1109/ICOCN.2017.8121297.

[32] J. O. Roa, A. R. Jiménez, F. Seco, J. C. Prieto, J. Ealo, Optimal placement of sensors for trilateration: Regular lattices vs meta-heuristic solutions, in: R. Moreno Díaz, F. Pichler, A. Quesada Arencibia (Eds.), Computer Aided Systems Theory - EUROCAST 2007, Springer Berlin Heidelberg, Berlin, Heidelberg, 2007, pp. 780-787.

[33] D. Plets, S. Bastiaens, L. Martens, W. Joseph, An analysis of the impact of led tilt on visible light positioning accuracy, Electronics 8 (2019). doi:10.3390/electronics8040389. 
[34] E. Jeong, S. Yang, H. Kim, S. Han, Tilted receiver angle error compensated indoor positioning system based on visible light communication, Electronics Letters 49 (2013) 890-892. doi:10.1049/el.2013.1368.

[35] J. Kim, S. Yang, Y. Son, S. Han, High-resolution indoor positioning using light emitting diode visible light and camera image sensor, IET Optoelectronics 10 (2016) 184-192. doi:10.1049/iet-opt.2015.0073.

[36] B. Zhou, V. Lau, Q. Chen, Y. Cao, Simultaneous positioning and orientating for visible light communications: Algorithm design and performance analysis, IEEE Transactions on Vehicular Technology 67 (2018) 11790-11804. doi:10.1109/TVT.2018.2875044.

[37] Y. Almadani, M. Ijaz, S. Bastiaens, S. Rajbhandari, W. Joseph, D. Plets, An experimental analysis of the effect of reflections on the performance of visible light positioning systems in warehouses, in: 2019 IEEE 2nd British and Irish Conference on Optics and Photonics (BICOP), 2019, pp. 1-4.

[38] B. Neuwinger, U. Witkowski, U. Rückert, Ad-hoc communication and localization system for mobile robots, in: Advances in Robotics, Springer Berlin Heidelberg, Berlin, Heidelberg, 2009, pp. 220-229.

[39] K.-W. Lee, J.-B. Park, B.-H. Lee, Dynamic localization with hybrid trilateration for mobile robots in intelligent space, Intelligent Service Robotics 1 (2008) 221-235. doi:10.1007/s11370-007-0012-1.

[40] C. W. Chow, Y. Liu, C. H. Yeh, J. Y. Sung, Y. L. Liu, A practical in-home illumination consideration to reduce data rate fluctuation in visible light communication, IEEE Wireless Communications 22 (2015) 17-23. doi:10.1109/MWC.2015.7096280. 